\title{
新的席夫碱型鹅去氧胆酸分子钳的微波无溶剂合成
}

\author{
石治川赵志刚* 李晖谭畑 \\ (西南民族大学化学与环境保护工程学院 成都 610041)
}

\begin{abstract}
摘要 在微波辐射无溶剂条件下, 以鹅去氧胆酸甲酯为隔离剂, 通过氨基与不同芳香醛缩合合成了十个含席夫碱结构 单元为手臂的新型鹅去氧胆酸分子钳. 通过微波法和常规法的对比发现，使用微波法后，产率从 $30 \% \sim 75 \%$ 提高到 $85 \% \sim 94 \%$, 反应时间从 $300 \sim 480 \mathrm{~min}$ 缩短到 $0.5 \sim 1.5 \mathrm{~min}$. 所有合成化合物的结构均经 ${ }^{1} \mathrm{H}$ NMR, IR, ESI-MS 和元素分 析确证. 利用紫外光谱滴定法考察了其对分子的识别性能研究. 实验结果表明, 这类分子钳人工受体对有机小分子具 有良好的识别能力.
\end{abstract}

关键词 我去氧胆酸; 分子钳; 微波合成; 分子识别

\section{Solvent-Free Synthesis of Novel Schiff Base Type Molecular Tweezers Based on Chenodeoxycholic Acid Promoted by Microwave Irradiation}

\author{
Shi, Zhichuan $\quad$ Zhao, Zhigang* Li, Hui $\quad$ Tan, Jiong \\ (College of Chemistry and Environmental Protection Engineering, Southwest University for Nationalities, Chengdu 610041)
}

\begin{abstract}
Ten new steroidal molecular tweezers were efficiently synthesized via a method employing microwave irradiation by using chenodeoxycholic acid as spacer and Schiff base unit as arm through the condensation of amines and different aromatic aldehydes. Compared with a conventional method, the yields were increased from $30 \% \sim 75 \%$ to $85 \% \sim 94 \%$ and the reaction times were reduced from $300 \sim 480 \mathrm{~min}$ to $0.5 \sim 1.5 \mathrm{~min}$. The structures of these novel molecular tweezers were characterized by ${ }^{1} \mathrm{H}$ NMR, IR, ESI-MS techniques and elemental analysis. The recognition properties of these molecular tweezers for organic molecules were investigated by UV-vis spectra. The results indicated that this type of molecular tweezers has good binding properties for organic molecules.
\end{abstract}

Keywords chenodeoxycholic acid; molecular tweezers; microwave synthesis; molecular recognition

非共价键相互作用在分子识别、分子自组装、化学 传输和仿生酶催化过程以及控制化合物形状中扮演着 重要作用 ${ }^{[1 ~ 2]}$. 利用合成的人工受体与适当底物之间的 分子识别以建立仿生仿酶模型的研究, 已成为生物有机 化学前沿的热点研究领域之一. 近年来, 分子钳作为一 类新型人工受体, 在分子器件、生物传感、分子识别等 领域显示出广阔的应用前景 ${ }^{[3 \sim 5]}$. 这类受体的主要结构 特征是坚硬的分子骨架做隔离基，连接不同大小、不同 种类的芳(杂)环构成刚性受体; 它开放性的空腔结构能 够使两个手臂与客体分子通过非共价键相互作用形成 识别过程. 在 “钳形” 分子中, 通常需要其两个手臂间
隔保持水平对齐, 并且有效距离在 $7 \AA^{[6,7]}$ 左右才能达到 很好的非共价键相互作用. 所以不会有太多已知结构化 合物能够被用做隔离基 ${ }^{[8,9]}$. 而鹅去氧胆酸由于具有大 而刚性的疏水凹面骨架及固有的不对称性， 3,7 位的羟 基处于相互平行状态，并且其直线距离在 $7 \AA$ 以内, 因 此是一种构筑分子钳人工受体的理想结构单元.

此外，微波技术特别是微波无溶剂技术为有机合成 提供了一个独特和环境友好的合成过程. 同传统合成方 法相比，具有操作简单、反应时间短、效率高、能耗低 等优点, 是绿色化学的一个重要组成部分 ${ }^{[10 ~ 19]}$. 纵观国 内外文献报道，合成含有席夫碱结构的我去氧胆酸分子

\footnotetext{
*E-mail: zzg63129@163.com

Received September 1, 2013; revised November 1, 2013; published online November 14, 2013.

Project supported by the Science and Technology Department of Sichuan Province (No. 2012SZ0160) and the Fundamental Research Funds of Central Universities, Southwest University for Nationalities (No. 13NZYQN12).

四川省科技支撑计划基金(No. 2012SZ0160)和西南民族大学中央高校基本科研业务费科学基金(No. 13NZYQN12)资助项目.
} 
钳还鲜见报道. 为了进一步拓展胆甾类分子钳的合成与 识别性能研究, 结合我们课题组在微波无溶剂合成方面 的研究基础 ${ }^{[20 ~ 22]}$, 本文在微波辐射条件下, 以鹅去氧胆 酸甲酯为隔离基, $3 \alpha, 7 \alpha$ 位通过酯键连接对硝基苯甲酰 氯, 然后用 $\mathrm{SnCl}_{2} \cdot 2 \mathrm{H}_{2} \mathrm{O}$ 将硝基还原为氨基, 最后在微波 无溶剂条件下使氨基与各种芳醛反应，合成席夫碱型鹅 去氧胆酸分子钳. 合成路线见 Scheme 1.

\section{1 实验部分}

\section{1 仪器与试剂}

核磁共振仪: Varian INOVA-400 $\mathrm{MHz}, \mathrm{Me}_{4} \mathrm{Si}$ 作为 内标, $\mathrm{CDCl}_{3}$ 为溶剂; 质谱仪: FINNIGAN-LCQ ${ }^{\mathrm{DECA}}$ 型; 红外光谱仪: PERKINEL-MER 1700 型, $\mathrm{KBr}$ 压片; 紫外 可见光谱仪: 北京普析 TU-1901 型分光光度计; 元素分 析仪: Carlo-Erba-1106 型; 微波反应器: 北京祥鹄科技 公司商用微波反应器 $\mathrm{XH}-100 \mathrm{~A}$; 旋光仪: WZZ-2B 自动 旋光仪; 熔点仪: X-4 型数字显示显微熔点仪. 所用试剂 均为市售化学纯或分析纯. 无水 $\mathrm{CH}_{2} \mathrm{Cl}_{2}$ ：加 $\mathrm{CaH}_{2}$ 回流 数天后, 反应前新蒸使用; 无水 $\mathrm{DMF}(N, N$-二甲基甲酰 胺): 加 $\mathrm{CaH}_{2}$ 干燥 $48 \mathrm{~h}$ 后, 减压蒸馏; $\mathrm{SOCl}_{2}$ : 重新蒸馏, 收集 $73 \sim 75{ }^{\circ} \mathrm{C}$ 馏分; 我去氧胆酸: Acros 公司进口. 中 间体 $3 \alpha, 7 \alpha$-二羟基- 5 3 -24-胆烷酸甲酯(2)按文献[23]的
方法合成.

1.2 中间体 $3 \alpha, 7 \alpha$-二(4-硝基苯甲酰氧基)-5 $\beta$-24-胆烷 酸甲酯(3)的微波合成与表征

在 $25 \mathrm{~mL}$ 圆底烧瓶中, 依次加入 $1 \mathrm{mmol}$ 我去氧胆 酸甲酯 2, $4 \mathrm{mmol}$ 新制的对硝基苯甲酰氯, $2.5 \mathrm{~g}$ 固体 $\mathrm{K}_{2} \mathrm{CO}_{3}, 0.4 \mathrm{~mL}$ 三乙胺, 充分搅拌混合均匀后, 将圆底烧 瓶置于微波炉中，并外接干燥管，于 $500 \mathrm{~W}$ 间歇式微波 辐射反应 $6 \mathrm{~min}, \mathrm{TLC}$ 监测反应进程, 反应结束后, 用 $\mathrm{CH}_{2} \mathrm{Cl}_{2}$ 提取 $(10 \mathrm{~mL} \times 2)$, 提取液分别用 $5 \% \mathrm{HCl}$ $(10 \mathrm{~mL} \times 3)$, 饱和食盐水溶液 $(10 \mathrm{~mL} \times 3)$ 洗涤, 无水 $\mathrm{Na}_{2} \mathrm{SO}_{4}$ 干燥, 过滤, 减压蒸去溶剂后得粗品, 硅胶柱层 析分离纯化[固定相用硅胶 $\mathrm{H}$, 洗脱剂为 $V\left(\mathrm{CH}_{2} \mathrm{Cl}_{2}\right)$ : $V\left(\mathrm{CH}_{3} \mathrm{COOC}_{2} \mathrm{H}_{5}\right)=50 ： 1$ ] 得到中间体 3. 淡黄色晶体, 产率 $88 \%$. m.p. $168 \sim 170{ }^{\circ} \mathrm{C} ;[\alpha]_{\mathrm{D}}^{20}-41.0$ (c 0.13 , $\left.\mathrm{CH}_{2} \mathrm{Cl}_{2}\right) ;{ }^{1} \mathrm{H} \mathrm{NMR}\left(\mathrm{CDCl}_{3}, 400 \mathrm{MHz}\right) \delta: 8.29 \sim 8.06(\mathrm{~m}$, $8 \mathrm{H}, \mathrm{ArH}), 5.21(\mathrm{~s}, 1 \mathrm{H}, 7 \beta-\mathrm{H}), 4.90 \sim 4.85(\mathrm{~m}, 1 \mathrm{H}, 3 \beta-\mathrm{H})$, $3.63\left(\mathrm{~s}, 3 \mathrm{H}, \mathrm{COOCH}_{3}\right), 1.04$ (s, 3H, 19- $\left.\mathrm{CH}_{3}\right), 0.94$ (d, $J=$ $\left.6.4 \mathrm{~Hz}, 3 \mathrm{H}, 21-\mathrm{CH}_{3}\right), 0.71\left(\mathrm{~s}, 3 \mathrm{H}, 18-\mathrm{CH}_{3}\right)$; IR (KBr) v: 2942, 2875, 1718, 1603, 1528, 1458, $1260 \mathrm{~cm}^{-1}$; ESI-MS $\mathrm{m} / \mathrm{z}(\%): 727\left[(\mathrm{M}+\mathrm{Na})^{+}, 100\right]$; Anal. calcd for $\mathrm{C}_{39} \mathrm{H}_{48^{-}}$ $\mathrm{N}_{2} \mathrm{O}_{10}$ : C 66.46, H 6.86, N 3.97; found C 66.57, H 6.88, N 3.95 .
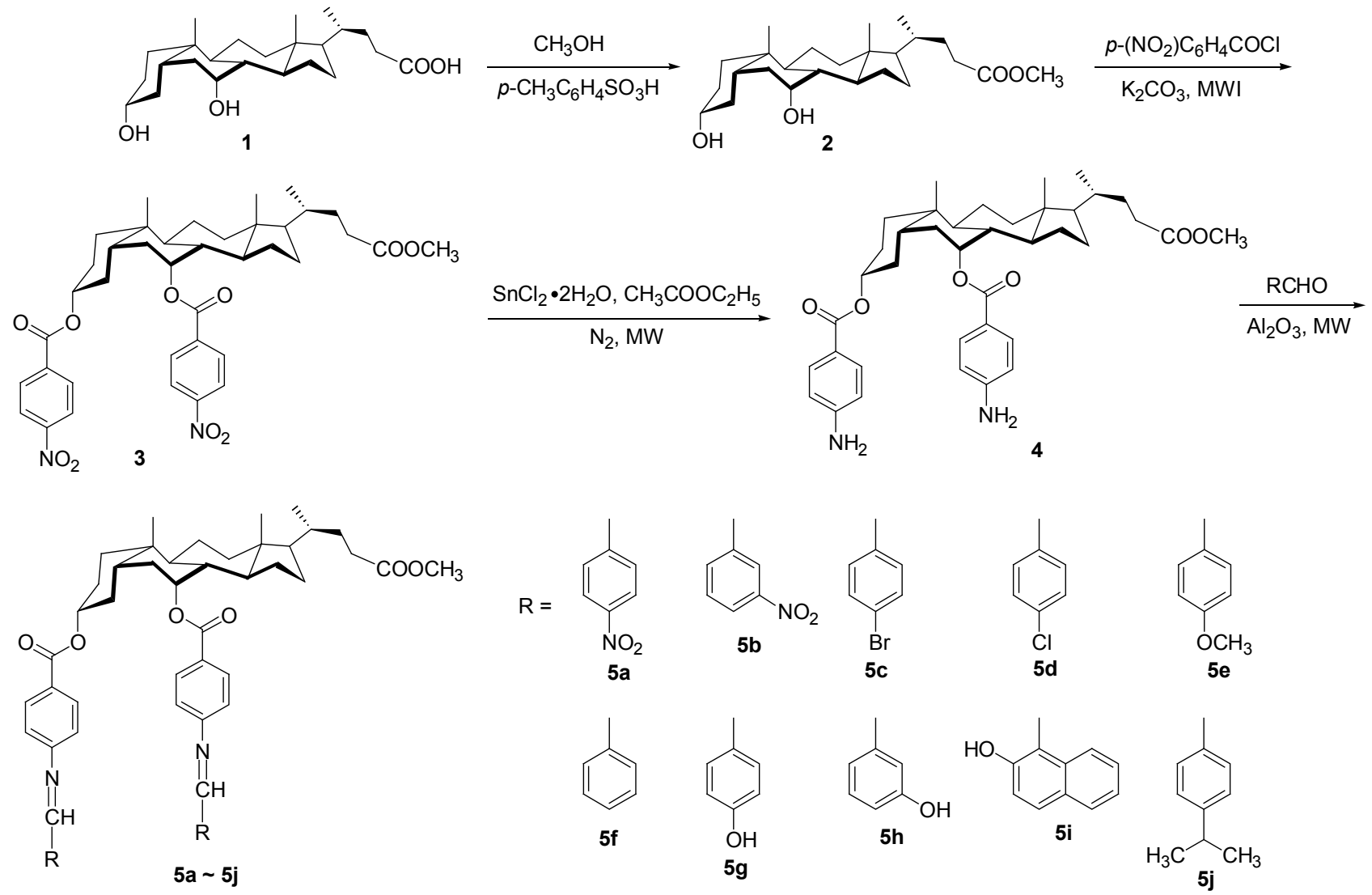

Scheme 1 


\section{3 中间体 $3 \alpha, 7 \alpha$-二(4-氨基苯甲酰氧基)- $5 \beta$ - 24 -胆烷} 酸甲酯(4)的微波合成与表征

在 $50 \mathrm{~mL}$ 三颈瓶中加入 $1 \mathrm{mmol}$ 中间体 $3,20 \mathrm{~mL}$ 乙 酸乙酯, $6 \mathrm{mmol}$ 的 $\mathrm{SnCl}_{2} \cdot 2 \mathrm{H}_{2} \mathrm{O}$, 在 $\mathrm{N}_{2}$ 保护下, 于 $200 \mathrm{~W}$ 微波辐射反应 $10 \mathrm{~min}, \mathrm{TLC}$ 监测反应进程, 反应结束后, 将混合物倾入 $30 \mathrm{~mL}$ 饱和 $\mathrm{NaHCO}_{3}$ 水溶液中, 振荡, 静 置, 分出有机层, 水层再用乙酸乙酯 $(15 \mathrm{~mL} \times 3)$ 提取, 合并滤液, 用饱和食盐水 $(15 \mathrm{~mL} \times 3)$ 洗涤, 无水 $\mathrm{Na}_{2} \mathrm{SO}_{4}$ 干燥, 过滤, 减压蒸去溶剂, 柱层析分离纯化[固定相用 硅胶 $\mathrm{H}$, 洗脱剂为 $\left.V\left(\mathrm{CH}_{2} \mathrm{Cl}_{2}\right): V\left(\mathrm{CH}_{3} \mathrm{COOC}_{2} \mathrm{H}_{5}\right)=5: 1\right]$ 得到中间体 4. 淡黄色晶体, 产率 90\%. m.p. 104 105 ${ }^{\circ} \mathrm{C} ;[\alpha]_{\mathrm{D}}^{20}-39.7\left(c 0.13, \mathrm{CH}_{2} \mathrm{Cl}_{2}\right) ;{ }^{1} \mathrm{H}$ NMR $\left(\mathrm{CDCl}_{3}, 400\right.$ MHz) $\delta: 8.07 \sim 6.78(\mathrm{~m}, 8 \mathrm{H}, \mathrm{ArH}), 5.48(\mathrm{~s}, 1 \mathrm{H}, 7 \beta-\mathrm{H})$, $4.90 \sim 4.86(\mathrm{~m}, 1 \mathrm{H}, 3 \beta-\mathrm{H}), 3.67\left(\mathrm{~s}, 3 \mathrm{H}, \mathrm{COOCH}_{3}\right), 3.51$ (brs, $4 \mathrm{H}, \mathrm{ArNH}_{2}$ ), 1.03 (s, 3H, 19- $\mathrm{CH}_{3}$ ), 0.97 (d, $J=6.4$ $\left.\mathrm{Hz}, 3 \mathrm{H}, 21-\mathrm{CH}_{3}\right), 0.68$ (s, 3H, 18- $\mathrm{CH}_{3}$ ); IR (KBr) v: 3479, 2963, 2877, 1716, 1598, 1501, 1477, $1230 \mathrm{~cm}^{-1}$; ESI-MS $m / z$ (\%): $667\left[(\mathrm{M}+\mathrm{Na})^{+}, 100\right]$. Anal. calcd for $\mathrm{C}_{39} \mathrm{H}_{52}{ }^{-}$ $\mathrm{N}_{2} \mathrm{O}_{6}$ : C 72.64, H 8.13, N 4.34; found C 72.85, H 8.12, N 4.35 .

\section{4 分子钳 $5 a \sim 5 j$ 的微波合成与表征}

在 $25 \mathrm{~mL}$ 圆底烧瓶中, 加入 $0.2 \mathrm{~g}(0.31 \mathrm{mmol})$ 的中 间体 $4,1 \mathrm{~g}$ 中性 $\mathrm{Al}_{2} \mathrm{O}_{3}, 0.65 \mathrm{mmol}$ 芳香醛, 充分混合均匀 后, 将圆底烧瓶置于微波炉中, 于 $300 \mathrm{~W}$ 微波辐射反应 $30 \sim 90 \mathrm{~s}, \mathrm{TLC}$ 监测反应进程, 反应结束后, 用 $\mathrm{CH}_{2} \mathrm{Cl}_{2}$ 提取 $(10 \mathrm{~mL} \times 3)$, 提取液减压蒸去溶剂后得粗品, 重结 晶得到纯品.

分子钳 $3 \alpha, 7 \alpha$-二[4-(4-硝基苯甲亚胺)苯甲酰氧 基]-5 $\beta$-24-胆烷酸甲酯(5a): 黄色晶体 $\left(\mathrm{CH}_{2} \mathrm{Cl}_{2}\right.$-石油醚重 结晶), 产率 $90 \%$. m.p. $184 \sim 186{ }^{\circ} \mathrm{C} ;[\alpha]_{\mathrm{D}}^{20}+172.3(c$ 1.3, $\left.\mathrm{CH}_{2} \mathrm{Cl}_{2}\right) ;{ }^{1} \mathrm{H}$ NMR $\left(\mathrm{CDCl}_{3}, 400 \mathrm{MHz}\right) \delta: 7.89(\mathrm{~s}, 1 \mathrm{H}$, NCHAr $), 7.87(\mathrm{~s}, 1 \mathrm{H}, \mathrm{NCHAr}), 7.81 \sim 6.60(\mathrm{~m}, 16 \mathrm{H}$, ArH), $5.15(\mathrm{~s}, 1 \mathrm{H}, 7 \beta-\mathrm{H}), 4.81 \sim 4.75(\mathrm{~m}, 1 \mathrm{H}, 3 \beta-\mathrm{H}), 3.64$ (s, $\left.3 \mathrm{H}, \mathrm{COOCH}_{3}\right), 0.99$ (s, 3H, 19-CH $\mathrm{CH}_{3}, 0.91$ (d, $J=6.4$ $\left.\mathrm{Hz}, 3 \mathrm{H}, 21-\mathrm{CH}_{3}\right), 0.68$ (s, 3H, 18- $\mathrm{CH}_{3}$ ); IR (KBr) v: 3445, 2938, 2848, 1702, 1641, 1523, 1238, 1125, $1092 \mathrm{~cm}^{-1}$; ESI-MS $m / z$ (\%): 934 [(M+Na) $\left.{ }^{+}, 100\right]$. Anal. calcd for $\mathrm{C}_{53} \mathrm{H}_{58} \mathrm{~N}_{4} \mathrm{O}_{10}$ : C 69.87, $\mathrm{H}$ 6.42, N 6.15; found $\mathrm{C} 69.94, \mathrm{H}$ $6.43, \mathrm{~N} 6.16$.

分子钳 $3 \alpha, 7 \alpha$-二[4-(3-硝基苯甲亚胺)苯甲酰氧 基]-5 $\beta$-24-胆烷酸甲酯 $(\mathbf{5 b})$ : 黄色晶体 $\left(\mathrm{CH}_{2} \mathrm{Cl}_{2}\right.$-乙醇重结 晶), 产率 $85 \%$. m.p. $151 \sim 153{ }^{\circ} \mathrm{C} ;[\alpha]_{\mathrm{D}}^{20}+272.3$ (c 1.2, $\left.\mathrm{CH}_{2} \mathrm{Cl}_{2}\right) ;{ }^{1} \mathrm{H}$ NMR $\left(\mathrm{CDCl}_{3}, 400 \mathrm{MHz}\right) \delta: 8.80(\mathrm{~s}, 1 \mathrm{H}$, NCHAr), $8.78(\mathrm{~s}, 1 \mathrm{H}, \mathrm{NCHAr}), 8.68 \sim 6.62(\mathrm{~m}, 16 \mathrm{H}$,

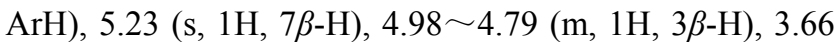
(s, $\left.3 \mathrm{H}, \mathrm{COOCH}_{3}\right), 0.97\left(\mathrm{~s}, 3 \mathrm{H}, 19-\mathrm{CH}_{3}\right), 0.93$ (d, $J=5.6$ $\left.\mathrm{Hz}, 3 \mathrm{H}, 21-\mathrm{CH}_{3}\right), 0.67$ (s, 3H, 18- $\mathrm{CH}_{3}$ ); IR (KBr) v: 3438, 2917, 2848, 1717, 1530, 1521, 1273, 1176, $1107 \mathrm{~cm}^{-1}$; ESI-MS $m / z(\%): 1845\left[(2 \mathrm{M}+\mathrm{Na})^{+}, 100\right]$. Anal. calcd for $\mathrm{C}_{53} \mathrm{H}_{58} \mathrm{~N}_{4} \mathrm{O}_{10}$ : C 69.87, H 6.42, N 6.15; found C 69.91, H 6.45, N 6.17.

分子钳 $3 \alpha, 7 \alpha$-二[4-(4-溴苯甲亚胺)苯甲酰氧基]- $5 \beta$ 24-胆烷酸甲酯 $(\mathbf{5 c})$ : 白色晶体 $\left(\mathrm{CH}_{2} \mathrm{Cl}_{2}\right.$-石油醚重结晶), 产率 94\%. m.p. $221 \sim 222{ }^{\circ} \mathrm{C} ;[\alpha]_{\mathrm{D}}^{20}+41.5$ (c 1.2 , $\left.\mathrm{CH}_{2} \mathrm{Cl}_{2}\right) ;{ }^{1} \mathrm{H}$ NMR $\left(\mathrm{CDCl}_{3}, 400 \mathrm{MHz}\right) \delta: 8.44(\mathrm{~s}, 1 \mathrm{H}$, NCHAr $), 8.41(\mathrm{~s}, 1 \mathrm{H}, \mathrm{NCHAr}), 8.39 \sim 6.65(\mathrm{~m}, 16 \mathrm{H}$, ArH), $5.22(\mathrm{~s}, 1 \mathrm{H}, 7 \beta-\mathrm{H}), 4.89 \sim 4.77(\mathrm{~m}, 1 \mathrm{H}, 3 \beta-\mathrm{H}), 3.64$ (s, $\left.3 \mathrm{H}, \mathrm{COOCH}_{3}\right), 0.95\left(\mathrm{~s}, 3 \mathrm{H}, 19-\mathrm{CH}_{3}\right), 0.88(\mathrm{~d}, J=3.6$ $\left.\mathrm{Hz}, 3 \mathrm{H}, 21-\mathrm{CH}_{3}\right), 0.68$ (s, 3H, 18-- $\mathrm{CH}_{3}$ ); IR (KBr) v: 3458, 2938, 2876, 1704, 1628, 1565, 1273, 1163, $1099 \mathrm{~cm}^{-1}$; ESI-MS $m / z(\%): 979\left[(\mathrm{M}+\mathrm{H})^{+}, 100\right]$. Anal. calcd for $\mathrm{C}_{53} \mathrm{H}_{58} \mathrm{Br}_{2} \mathrm{~N}_{2} \mathrm{O}_{6}$ : C 65.03, H 5.97, N 2.86; found C 65.15, H $5.97, \mathrm{~N} 2.87$.

分子钳 $3 \alpha, 7 \alpha$-二[4-(4-氯苯甲亚胺)苯甲酰氧基]- $5 \beta$ 24-胆烷酸甲酯 $(\mathbf{5 d})$ : 白色晶体 $\left(\mathrm{CH}_{2} \mathrm{Cl}_{2}\right.$-石油醚重结晶), 产率 87\%. m.p. $200 \sim 201{ }^{\circ} \mathrm{C} ;[\alpha]_{\mathrm{D}}^{20}+186.3$ (c 1.5, $\left.\mathrm{CH}_{2} \mathrm{Cl}_{2}\right) ;{ }^{1} \mathrm{H}$ NMR $\left(\mathrm{CDCl}_{3}, 400 \mathrm{MHz}\right) \delta: 7.89(\mathrm{~s}, 1 \mathrm{H}$, NCHAr), $7.87(\mathrm{~s}, 1 \mathrm{H}, \mathrm{NCHAr}), 7.85 \sim 6.60(\mathrm{~m}, 16 \mathrm{H}$, $\mathrm{ArH}), 5.18(\mathrm{~s}, 1 \mathrm{H}, 7 \beta-\mathrm{H}), 4.85 \sim 4.74(\mathrm{~m}, 1 \mathrm{H}, 3 \beta-\mathrm{H}), 3.64$ (s, $\left.3 \mathrm{H}, \mathrm{COOCH}_{3}\right), 0.99$ (s, $\left.3 \mathrm{H}, 19-\mathrm{CH}_{3}\right), 0.91$ (d, $J=6.4$ $\mathrm{Hz}, 3 \mathrm{H}, 21-\mathrm{CH}_{3}$ ), 0.68 (s, 3H, 18- $\mathrm{CH}_{3}$ ); IR (KBr) v: 3445, 2945, 2862, 1704, 1594, 1531, 1287, 1169, $1110 \mathrm{~cm}^{-1}$; ESI-MS $m / z$ (\%): 913 [(M+Na $\left.)^{+}, 100\right]$. Anal. calcd for $\mathrm{C}_{53} \mathrm{H}_{58} \mathrm{Cl}_{2} \mathrm{~N}_{2} \mathrm{O}_{6}$ : C 71.53, H 6.57, N 3.15; found C 71.42, $\mathrm{H}$ $6.59, \mathrm{~N} 3.14$

分子钳 $3 \alpha, 7 \alpha$-二[4-(4-甲氧基苯甲亚胺)苯甲酰氧 基]-5 $\beta$-24-胆烷酸甲酯(5e): 黄色晶体 $\left(\mathrm{CH}_{2} \mathrm{Cl}_{2}\right.$-石油醚重 结晶), 产率 $85 \%$. m.p. $163 \sim 165{ }^{\circ} \mathrm{C}$; $[\alpha]_{\mathrm{D}}^{20}+157.6(c$ 1.7, $\left.\mathrm{CH}_{2} \mathrm{Cl}_{2}\right) ;{ }^{1} \mathrm{H} \mathrm{NMR}\left(\mathrm{CDCl}_{3}, 400 \mathrm{MHz}\right) \delta: 8.37(\mathrm{~s}, 1 \mathrm{H}$, NCHAr), $8.34(\mathrm{~s}, 1 \mathrm{H}, \mathrm{NCHAr}), 8.12 \sim 6.98(\mathrm{~m}, 16 \mathrm{H}$, ArH), 5.25 (s, 1H, 7 $\beta-\mathrm{H}), 5.02 \sim 4.97(\mathrm{~m}, 1 \mathrm{H}, 3 \beta-\mathrm{H}), 3.90$ $\left(\mathrm{s}, 3 \mathrm{H}, \mathrm{ArOCH}_{3}\right), 3.92\left(\mathrm{~s}, 3 \mathrm{H}, \mathrm{ArOCH}_{3}\right), 3.67(\mathrm{~s}, 3 \mathrm{H}$, $\left.\mathrm{COOCH}_{3}\right), 1.06\left(\mathrm{~s}, 3 \mathrm{H}, 19-\mathrm{CH}_{3}\right), 0.93(\mathrm{~d}, J=6.4 \mathrm{~Hz}, 3 \mathrm{H}$, $\left.21-\mathrm{CH}_{3}\right), 0.69$ (s, 3H, 18- $\left.\mathrm{CH}_{3}\right)$; IR ( $\left.\mathrm{KBr}\right) v: 3445,2924$, 2848, 1706, 1629, 1509, 1273, 1169, $1102 \mathrm{~cm}^{-1}$; ESI-MS $m / z$ (\%): $904\left[(\mathrm{M}+\mathrm{Na})^{+}, 100\right]$. Anal. calcd for $\mathrm{C}_{55} \mathrm{H}_{64} \mathrm{~N}_{2} \mathrm{O}_{8}$ : C 74.97, H 7.32, N 3.18; found C 75.02, H 7.33, N 3.17 .

分子钳 $3 \alpha, 7 \alpha$-二[4-(苯甲亚胺)苯甲酰氧基]-5 $\beta$-24- 
胆烷酸甲酯 $(\mathbf{5 f})$ : 淡黄色晶体 $\left(\mathrm{CH}_{2} \mathrm{Cl}_{2}\right.$ - 石油醚重结晶), 产率 90\%. m.p. $114 \sim 116{ }^{\circ} \mathrm{C}$; $[\alpha]_{\mathrm{D}}^{20}+101.2$ (c 1.0, $\left.\mathrm{CH}_{2} \mathrm{Cl}_{2}\right) ;{ }^{1} \mathrm{H} \mathrm{NMR}\left(\mathrm{CDCl}_{3}, 400 \mathrm{MHz}\right) \delta: 8.36(\mathrm{~s}, 1 \mathrm{H}$, NCHAr), 8.33 (s, 1H, NCHAr), 8.14 $6.61(\mathrm{~m}, 16 \mathrm{H}$, ArH), $5.23(\mathrm{~s}, 1 \mathrm{H}, 7 \beta-\mathrm{H}), 4.91 \sim 4.81(\mathrm{~m}, 1 \mathrm{H}, 3 \beta-\mathrm{H}), 3.64$ (s, 3H, $\left.\mathrm{COOCH}_{3}\right), 1.00$ (s, 3H, 19- $\left.\mathrm{CH}_{3}\right), 0.92$ (d, $J=6.4$ $\left.\mathrm{Hz}, 3 \mathrm{H}, 21-\mathrm{CH}_{3}\right), 0.69$ (s, 3H, 18- $\left.\mathrm{CH}_{3}\right)$; IR ( $\left.\mathrm{KBr}\right)$ v: 3375, 2931, 2862, 1704, 1600, 1516, 1273, 1169, $1106 \mathrm{~cm}^{-1}$; ESI-MS $m / z(\%): 1663$ [(2M+Na) $\left.{ }^{+}, 100\right]$. Anal. calcd for $\mathrm{C}_{53} \mathrm{H}_{60} \mathrm{~N}_{2} \mathrm{O}_{6}$ : C 77.53, H 7.37, N 3.41; found C 77.50, H 7.37, N 3.43 .

分子钳 $3 \alpha, 7 \alpha$-二[4-(4-羟基苯甲亚胺)苯甲酰氧 基]-5 $\beta$-24-胆烷酸甲酯 $(\mathbf{5 g})$ : 黄色晶体 $\left(\mathrm{CH}_{2} \mathrm{Cl}_{2}\right.$-石油醚重 结晶), 产率 $90 \%$. m.p. $65 \sim 67{ }^{\circ} \mathrm{C}$; $[\alpha]_{\mathrm{D}}^{20}+90.2$ (c 1.4, $\left.\mathrm{CH}_{2} \mathrm{Cl}_{2}\right) ;{ }^{1} \mathrm{H}$ NMR $\left(\mathrm{CDCl}_{3}, 400 \mathrm{MHz}\right) \delta: 8.31(\mathrm{~s}, 1 \mathrm{H}$, NCHAr), $8.23(\mathrm{~s}, 1 \mathrm{H}, \mathrm{NCHAr}), 7.95 \sim 6.61(\mathrm{~m}, 16 \mathrm{H}$, ArH), $5.30(\mathrm{~s}, 1 \mathrm{H}, 7 \beta-\mathrm{H}), 4.95 \sim 4.80(\mathrm{~m}, 1 \mathrm{H}, 3 \beta-\mathrm{H}), 3.62$ (s, $\left.3 \mathrm{H}, \mathrm{COOCH}_{3}\right), 0.97$ (s, 3H, 19- $\left.\mathrm{CH}_{3}\right), 0.91$ (d, $J=6.0$ $\left.\mathrm{Hz}, 3 \mathrm{H}, 21-\mathrm{CH}_{3}\right), 0.79$ (s, 3H, 18- $\mathrm{CH}_{3}$ ); IR (KBr) v: 3431, 2924, 2848, 1704, 1620, 1447, 1267, 1156, $1092 \mathrm{~cm}^{-1}$; ESI-MS $m / z(\%): 853\left[(\mathrm{M}+\mathrm{H})^{+}, 100\right]$. Anal. calcd for $\mathrm{C}_{53} \mathrm{H}_{60} \mathrm{~N}_{2} \mathrm{O}_{8}$ : C 74.62, H 7.09, N 3.28; found C 74.68, H 7.07, N 3.26.

分子钳 $3 \alpha, 7 \alpha$-二[4-(3-羟基苯甲亚胺)苯甲酰氧 基]-5 $\beta$-24-胆烷酸甲酯 $(\mathbf{5 h})$ : 黄色晶体 $\left(\mathrm{CH}_{2} \mathrm{Cl}_{2}\right.$-石油醚重 结晶), 产率 $88 \%$. m.p. $85 \sim 86{ }^{\circ} \mathrm{C} ;[\alpha]_{\mathrm{D}}^{20}+110.1$ (c 1.0, $\left.\mathrm{CH}_{2} \mathrm{Cl}_{2}\right) ;{ }^{1} \mathrm{H} \mathrm{NMR}\left(\mathrm{CDCl}_{3}, 400 \mathrm{MHz}\right) \delta: 8.35(\mathrm{~s}, 1 \mathrm{H}$, NCHAr), $8.31(\mathrm{~s}, 1 \mathrm{H}, \mathrm{NCHAr}), 8.20 \sim 6.62(\mathrm{~m}, 16 \mathrm{H}$, ArH), $5.31(\mathrm{~s}, 1 \mathrm{H}, 7 \beta-\mathrm{H}), 4.96 \sim 4.80(\mathrm{~m}, 1 \mathrm{H}, 3 \beta-\mathrm{H}), 3.63$ (s, $\left.3 \mathrm{H}, \mathrm{COOCH}_{3}\right), 0.96$ (s, 3H, 19- $\left.\mathrm{CH}_{3}\right), 0.93$ (d, $J=6.0$ $\left.\mathrm{Hz}, 3 \mathrm{H}, 21-\mathrm{CH}_{3}\right), 0.79$ (s, 3H, 18- $\mathrm{CH}_{3}$ ); IR (KBr) v: 3428, 2924, 2848, 1704, 1634, 1493, 1217, 1160, $1062 \mathrm{~cm}^{-1}$; ESI-MS $m / z(\%): 853\left[(\mathrm{M}+\mathrm{H})^{+}, 100\right]$. Anal. calcd for $\mathrm{C}_{53} \mathrm{H}_{60} \mathrm{~N}_{2} \mathrm{O}_{8}$ : C 74.62, H 7.09, N 3.28; found C 74.63, H $7.07, \mathrm{~N} 3.29$

分子钳 $3 \alpha, 7 \alpha$-二[4-(2-羟基奈甲亚胺)苯甲酰氧 基]- $5 \beta$-24-胆烷酸甲酯(5i): 黄色晶体(乙醇重结晶), 产 率 85\%. m.p. $108 \sim 110{ }^{\circ} \mathrm{C}$; $[\alpha]_{\mathrm{D}}^{20}+251.2$ (c 1.3, $\left.\mathrm{CH}_{2} \mathrm{Cl}_{2}\right) ;{ }^{1} \mathrm{H}$ NMR $\left(\mathrm{CDCl}_{3}, 400 \mathrm{MHz}\right) \delta: 8.20(\mathrm{~s}, 1 \mathrm{H}$, NCHAr), $8.12(\mathrm{~s}, 1 \mathrm{H}, \mathrm{NCHAr}), 8.08 \sim 6.62(\mathrm{~m}, 20 \mathrm{H}$, ArH), $5.31(\mathrm{~s}, 1 \mathrm{H}, 7 \beta-\mathrm{H}), 4.97 \sim 4.81(\mathrm{~m}, 1 \mathrm{H}, 3 \beta-\mathrm{H}), 3.66$ (s, $\left.3 \mathrm{H}, \mathrm{COOCH}_{3}\right), 0.98$ (s, 3H, 19-CH $\mathrm{CH}_{3}, 0.94$ (d, $J=6.4$ $\left.\mathrm{Hz}, 3 \mathrm{H}, 21-\mathrm{CH}_{3}\right), 0.79$ (s, 3H, 18- $\left.\mathrm{CH}_{3}\right)$; IR (KBr) v: 3445, 2917, 2848, 1717, 1634, 1572, 1273, 1176, $1106 \mathrm{~cm}^{-1}$; ESI-MS $m / z(\%): 953\left[(\mathrm{M}+\mathrm{H})^{+}, 100\right]$. Anal. calcd for
$\mathrm{C}_{61} \mathrm{H}_{64} \mathrm{~N}_{2} \mathrm{O}_{8}$ : C 76.86, H 6.77, N 2.94; found $\mathrm{C}$ 76.92, $\mathrm{H}$ 6.76, N 2.93 .

分子钳 $3 \alpha, 7 \alpha$-二[4-(4-异丙基苯甲亚胺)苯甲酰氧 基]-5 $\beta$-24-胆烷酸甲酯 $(\mathbf{5 j})$ : 黄色晶体 $\left(\mathrm{CH}_{2} \mathrm{Cl}_{2}\right.$-石油醚重 结晶), 产率 $90 \%$. m.p. 202 204 ${ }^{\circ} \mathrm{C}$; $[\alpha]_{\mathrm{D}}^{20}+172.5$ (c $\left.1.4, \mathrm{CH}_{2} \mathrm{Cl}_{2}\right) ;{ }^{1} \mathrm{H}$ NMR $\left(\mathrm{CDCl}_{3}, 400 \mathrm{MHz}\right) \delta: 8.24(\mathrm{~s}, 1 \mathrm{H}$, NCHAr), $8.20(\mathrm{~s}, 1 \mathrm{H}, \mathrm{NCHAr}), 8.10 \sim 6.51(\mathrm{~m}, 16 \mathrm{H}$, ArH), 5.21 (s, 1H, $7 \beta-\mathrm{H}), 4.92 \sim 4.79(\mathrm{~m}, 1 \mathrm{H}, 3 \beta-\mathrm{H}), 3.64$ (s, $\left.3 \mathrm{H}, \mathrm{COOCH}_{3}\right), 3.07$ (s, 6H, $\left.\mathrm{CH}_{3} \mathrm{NCH}_{3}\right), 2.93$ (s, 6H, $\left.\mathrm{CH}_{3} \mathrm{NCH}_{3}\right), 1.01$ (s, 3H, 19- $\left.\mathrm{CH}_{3}\right), 0.93(\mathrm{~d}, J=6.4 \mathrm{~Hz}, 3 \mathrm{H}$, $\left.21-\mathrm{CH}_{3}\right), 0.70\left(\mathrm{~s}, 3 \mathrm{H}, 18-\mathrm{CH}_{3}\right)$; IR ( $\left.\mathrm{KBr}\right) v: 3445,2931$, 2862, 1710, 1586, 1537, 1280, 1163, $1108 \mathrm{~cm}^{-1}$; ESI-MS $m / z(\%): 905\left[(\mathrm{M}+\mathrm{H})^{+}, 100\right]$. Anal. calcd for $\mathrm{C}_{59} \mathrm{H}_{72} \mathrm{~N}_{2} \mathrm{O}_{6}$ : C 78.28, H 8.02, N 3.09; found C 78.30, H 8.01, N 3.10.

\section{5 分子钳 $5 a \sim 5 j$ 的常规合成通法}

将 $0.2 \mathrm{~g}(0.31 \mathrm{mmol})$ 的中间体 4 溶于 $40 \mathrm{~mL}$ 乙醇中, 加入芳香醛 $0.65 \mathrm{mmol}$, 回流反应 $5 \sim 8 \mathrm{~h}$. 薄层色谱 (TLC)监测反应完成后, 减压下蒸除溶剂后得粗品, 重 结晶得到纯品，产率在 30\% 75\%之间.

\section{6 识别性能测试}

以无水氯仿作溶剂, 固定主体分子钳的浓度在 $2 \times$ $10^{-5} \sim 10 \times 10^{-5} \mathrm{~mol} / \mathrm{L}$ 范围内(由主体摩尔消光系数的大 小决定所配溶液浓度的大小), 加入不同浓度的客体芳 胺, 客体浓度在 $10^{-4} \sim 10^{-5} \mathrm{~mol} / \mathrm{L}$. 随着客体的不断加 入，主客体充分作用达到平衡后. 测定各组配合物溶液 的吸光度值, 为消除客体化合物在紫外区的干扰, 采用 相同浓度的客体氯仿液作参比, 同时为排除体积变化对 吸光度的影响, 主体中加入客体化合物的总体积不超过 $100 \mu \mathrm{L}$. 测定温度控制在 $(25 \pm 0.1){ }^{\circ} \mathrm{C}$, 吸光度误差不超 过 \pm 0.002 .

\section{2 结果与讨论}

\section{1 影响反应的因素及条件选择}

在实验过程中, 为了优化反应条件, 以分子钳 $5 \mathbf{a}$ 为 合成例子，详细考察了微波功率，辐射时间，载体种类， 以及常规溶剂条件对反应的影响, 综合几种影响因素, 得到了较佳的反应条件(表 1 ).

\section{2 微波法与常规法合成目标物 $5 a \sim 5 j$ 的对比}

两种不同的加热方法合成目标物 $\mathbf{5 a} \sim \mathbf{5 j}$ 的反应时 间和产率列于表 2. 从实验结果可以看出，在微波辐射 无溶剂条件下，以优良产率得到了 10 个新的席夫碱分 子钳. 微波法与传统加热合成方法相比, 具有以下优势: (1)反应速率增大了 200 600 倍，大大缩短了反应时间; (2)不受溶剂沸点，挥发性等因素影响，常规法所需溶剂 
表 1 化合物 $5 \mathbf{a}$ 的优化反应条件研究

Table 1 Optimization of the reaction conditions synthesizing compound $\mathbf{5 a}$

\begin{tabular}{|c|c|c|c|c|c|}
\hline Entry & Solvent & Supporter & Time/min & Power/W & Yield/\% \\
\hline 1 & EtOH & - & 300 & - & $62^{a}$ \\
\hline 2 & $\mathrm{MeOH}$ & - & 300 & - & $52^{a}$ \\
\hline 3 & DMF & - & 300 & - & $30^{a}$ \\
\hline 4 & DMSO & - & 300 & - & $32^{a}$ \\
\hline 5 & - & Silica gel H & 1.5 & 300 & $40^{b}$ \\
\hline 6 & - & Neutral $\mathrm{Al}_{2} \mathrm{O}_{3}$ & 1.5 & 300 & $90^{b}$ \\
\hline 7 & - & $\mathrm{K}_{2} \mathrm{CO}_{3}$ & 1.5 & 300 & $72^{b}$ \\
\hline 8 & - & Alkaline $\mathrm{Al}_{2} \mathrm{O}_{3}$ & 1.5 & 300 & $81^{b}$ \\
\hline 9 & - & Zeolite & 1.5 & 300 & $61^{b}$ \\
\hline 10 & - & Neutral $\mathrm{Al}_{2} \mathrm{O}_{3}$ & 1.0 & 300 & $67^{b}$ \\
\hline 11 & - & Neutral $\mathrm{Al}_{2} \mathrm{O}_{3}$ & 2.0 & 300 & $78^{b}$ \\
\hline 12 & - & Neutral $\mathrm{Al}_{2} \mathrm{O}_{3}$ & 1.5 & 250 & $80^{b}$ \\
\hline 13 & - & Neutral $\mathrm{Al}_{2} \mathrm{O}_{3}$ & 1.5 & 350 & $85^{b}$ \\
\hline
\end{tabular}

表 2 微波法与常规法合成目标物 $\mathbf{5 a} \sim 5 \mathbf{j}$ 的比较

Table 2 Synthesis comparison of between microwave irradiation and conventional of compounds $\mathbf{5 a} \sim \mathbf{5 j}$

\begin{tabular}{|c|c|c|c|c|c|}
\hline \multirow{2}{*}{ Comp. } & \multicolumn{2}{|c|}{ Conventional } & \multicolumn{2}{|c|}{ Microwave } & \multirow{2}{*}{$T_{\mathrm{C}}{ }^{a} / T_{\mathrm{MWI}}{ }^{b}$} \\
\hline & Time/min & Yield/\% & Time/min & Yield/\% & \\
\hline $5 a$ & 300 & 62 & 1.5 & 90 & 200 \\
\hline $5 b$ & 360 & 30 & 1.5 & 85 & 240 \\
\hline $5 c$ & 420 & 64 & 1.0 & 94 & 420 \\
\hline $5 d$ & 300 & 69 & 0.5 & 87 & 600 \\
\hline $5 e$ & 480 & 75 & 1.5 & 85 & 320 \\
\hline $5 f$ & 300 & 52 & 1.0 & 90 & 300 \\
\hline $5 g$ & 480 & 61 & 1.5 & 90 & 320 \\
\hline $5 \mathrm{~h}$ & 420 & 53 & 1.5 & 88 & 280 \\
\hline $5 \mathbf{i}$ & 360 & 70 & 1.5 & 85 & 240 \\
\hline $5 \mathbf{j}$ & 300 & 48 & 1.0 & 90 & 300 \\
\hline
\end{tabular}

均为 $40 \mathrm{~mL}$ 乙醇，而微波法则不使用任何溶剂，反应可 在较宽的温度范围内进行; (3)目标分子钳的产率得到较 大的提高，微波法的产率在 $85 \%$ \% 94\%之间，而传统加 热法的产率在 30\% 75\%之间, 这主要是因为微波加热 存在热.效应、过热现象和非热效应, 加之微波的介电加 热和内加热特性，使反应速度极大的提高. 由于微波反 应的时间短，因此副反应少，副产物少，故产率高; 而 常规加热时间较长，少则几个小时，多则几十个小时， 这样副反应多, 副产物多, 所以产率较低.

\section{3 目标化合物 $5 a \sim 5 j$ 的结构确证}

在 ${ }^{1} \mathrm{H}$ NMR 谱图中, $\delta 7.89 \sim 8.80$ 为席夫碱结构上的 NCHAr，而另外一组 NCHAr 在 $\delta 7.87 \sim 8.78$. 在核磁谱 图中, $\delta 0.95 \sim 1.06,0.68 \sim 0.79$ 两组单峰和 $0.88 \sim 0.94-$ 组双峰, 是我去氧胆酸的特征结构峰, $\delta 3.62 \sim 3.67$ 为载 体上羒酸甲酯的特征峰; 在 IR 谱图中, $1505 \sim 1546 \mathrm{~cm}^{-1}$ 附近出现的峰为席夫碱的 $\mathrm{C}=\mathrm{N}$ 吸收峰, 1717 1739 $\mathrm{cm}^{-1}$ 出现的是酯基中羰基的伸缩振动吸收峰; 从质谱 图中可见，化合物 $\mathbf{5 a} \sim 5 \mathbf{j}$ 均能给出分子离子峰，其 $m / z$ 与相对应分子式的分子量是一致的. 元素分析结果表 明，所有化合物的组成与其实验式相符. 综上所述，化 合物 $5 \mathbf{a} \sim 5 \mathbf{j}$ 所有光谱和元素分析数据与其结构式相符, 表明所合成的化合物为目标化合物.

\section{4 主客体配合物的形成及其化学计量}

在紫外光谱滴定实验中，固定主体分子钳 $(\mathrm{H})$ 浓度， 不断改变加入的客体 $(\mathrm{G})$ 的浓度，随着客体分子的不断 加入, 会引起主体的特征吸光度发生变化. 通常, 如果 主客体之间有识别配合作用, 形成了超分子配合物, 则 其特征吸光度呈规律性上升或规律性下降趋势, 表明客 体分子进入主体分子裂穴并产生了识别配合作用，典型 的吸收变化见图 1. 在 $[\mathrm{G}]_{0} \gg[\mathrm{H}]_{0}$ 时, 以 $1 /[\mathrm{G}]_{0}$ 对 $1 / \Delta A$ 作图, 均给出良好的线性关系, 以分子钳 $\mathbf{5 a}$ 为例, 结果 如图 2 所示. 表明芳胺与主体分子钳产生了识别配合作 用, 并形成了 $1: 1$ 型超分子配合物, 根据直线的截距和 斜率计算出配合物的结合常数 $\left(K_{\mathrm{a}}\right)$ 和自由能变化 $\left(-\Delta G^{0}\right)$ 列于表 3 .

主体 5a, 5d 5 $\mathrm{f}$ 对所考察的客体芳胺均具有良好的 识别能力, 主客体间形成 $1: 1$ 型超分子配合物. 主体 5e, 5 f 对客体的识别能力依次为: 苯胺 $>$ 邻甲氧基苯 胺 $>$ 对甲氧苯胺 $>$ 间甲氧苯胺, 而主体 $5 \mathrm{a}, 5 \mathrm{~d}$ 对客体的 识别能力为: 苯胺 $>$ 邻甲氧苯胺 $>$ 间甲氧苯胺 $>$ 对甲氧 苯胺, 并且它们均显示出对客体苯胺优良的选择性, 其

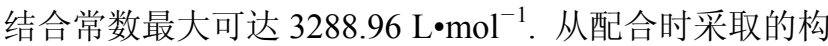
象来分析, 这种识别选择性的一种可能的解释是客体诱 导主体的几何互补有差异，即苯胺与主体分子的大小、 形状匹配和几何互补关系比邻甲氧基苯胺、对甲氧基苯



图 1 分子钳 $5 \mathbf{a}\left(2.80 \times 10^{-5} \mathrm{~mol} \cdot \mathrm{L}^{-1}\right)$ 中加入苯胺的 $\mathrm{UV}$-vis 谱 图

Figure 1 UV-vis spectra of molecular tweezers 5a $\left(2.80 \times 10^{-5}\right.$ $\left.\mathrm{mol} \cdot \mathrm{L}^{-1}\right)$ in the presence of aniline (a) 0 , (b) $0.56 \times 10^{-4}$, (c) $1.12 \times 10^{-4}$, (d) $1.68 \times 10^{-4}$, (e) $2.24 \times 10^{-4}$, (f) $2.80 \times 10^{-4}$, (g) $3.92 \times 10^{-4}$, (h) $4.48 \times 10^{-4} \mathrm{~mol} \cdot \mathrm{L}^{-1} \cdot \lambda_{\max }=247.0 \mathrm{~nm}$ 




图 $225{ }^{\circ} \mathrm{C}$ 时 $\mathrm{CHCl}_{3}$ 中分子钳 $\mathbf{5 a}$ 与苯胺形成配合物的 $1 / \Delta A$ 对 $1 /[\mathrm{G}]_{0}$ 作图

Figure 2 Typical plot of $1 / \Delta A$ versus $1 /[\mathrm{G}]_{0}$ for the inclusion complex of molecular tweezers $5 \mathbf{a}$ with aniline in $\mathrm{CHCl}_{3}$ at $25{ }^{\circ} \mathrm{C}$

表 $325{ }^{\circ} \mathrm{C}$ 时分子钳 $\mathbf{5 a}, \mathbf{5 d} \sim \mathbf{5 f}$ 与客体分子在 $\mathrm{CHCl}_{3}$ 中形成 包结配合物的结合常数 $\left(K_{\mathrm{a}}\right)$ 及自由能变化 $\left(-\Delta G^{0}\right)$

Table 3 Association constants $\left(K_{\mathrm{a}}\right)$ and Gibbs free energy changes $\left(-\Delta G^{0}\right)$ for the inclusion complexes of guest molecules with molecular tweezers $\mathbf{5 a}, \mathbf{5 d} \sim \mathbf{5 f}$ in $\mathrm{CHCl}_{3}$ at $25{ }^{\circ} \mathrm{C}$

\begin{tabular}{llcc}
\hline Host & Guest & $K_{\mathrm{a}} /\left(\mathrm{L} \cdot \mathrm{mol}^{-1}\right)$ & $-\Delta G^{0} /\left(\mathrm{kJ} \cdot \mathrm{mol}^{-1}\right)$ \\
\hline \multirow{4}{*}{ 5a } & Aniline & 1666.35 & 18.38 \\
& $o$-Methoxyaniline & 1634.18 & 18.33 \\
& $m$-Methoxyaniline & 708.58 & 16.26 \\
& $p$-Methoxyaniline & 459.34 & 15.19 \\
& Aniline & 3070.10 & 19.89 \\
5d & $o$-Methoxyaniline & 1207.12 & 17.58 \\
& $m$-Methoxyaniline & 751.53 & 16.41 \\
& $p$-Methoxyaniline & 593.23 & 15.82 \\
& Aniline & 2582.65 & 19.47 \\
5e & $o$-Methoxyaniline & 2478.56 & 19.36 \\
& $m$-Methoxyaniline & 847.78 & 16.71 \\
& $p$-Methoxyaniline & 1058.42 & 17.26 \\
& Aniline & 3288.96 & 20.06 \\
5f & $o$-Methoxyaniline & 2019.92 & 18.86 \\
& $m$-Methoxyaniline & 378.78 & 14.71 \\
& $p$-Methoxyaniline & 814.58 & 16.61 \\
\hline
\end{tabular}

胺和间甲氧基苯胺好, 形成配合物能量更低和稳定. 主 体 $5 e, 5 f$ 与苯胺、邻甲氧基苯胺、对甲氧基苯胺和间甲 氧基苯胺形成配合物的最低能量从小到大的顺序是


与客体分子的结合常数大小顺序为苯胺 $>$ 邻甲氧基苯 胺 $>$ 对甲氧基苯胺 $>$ 间甲氧基苯胺; 而主体 $\mathbf{5 a}, \mathbf{5 d}$ 与这 些客体分子形成的配合物的最低能量顺序则为 $E$ 莱脱 $<$

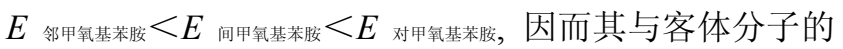
结合常数大小顺序则为苯胺 $>$ 邻甲氧基苯胺 $>$ 间甲氧 基苯胺 $>$ 对甲氧基苯胺. 主体 $5 \mathrm{a}, 5 \mathrm{5d}, 5 \mathrm{e}, \mathbf{5}$ 在同这四种 客体分子形成超分子配合物时, 识别模式均为客体氨基
与主体裂穴上部的酯氧形成两重氢键作用和主客体芳 环之间的 $\pi-\pi$ stacking 作用.

\section{3 结论}

在微波辐射无溶剂条件下, 发展了一种由鹅去氧胆 酸甲酯为隔离基, 中性三氧化二铝为载体, 通过氨基和 不同芳香醛缩合，快速高效合成鹅去氧胆酸分子钳的简 便方法，其产率可达 85\% 94\%. 该方法的显著优点是 反应时间短、产率高、操作简便、对环境友好. 同时通 过初步的识别性能研究，这类分子钳人工受体对芳胺分 子具有优良的识别配合性能.

\section{References}

[1] Ali, H.; Steven, T.; Gianluca, A.; Nicola, A.; Christopher, A. R.; Peter, D. W. B. J. Am. Chem. Soc. 2006, 128, 15903.

[2] Jeffrey, P. P.; Timothy, E. G. Org. Lett. 2006, 8, 2163.

[3] Hardouin-Lerouge, M.; Hudhomme, P.; Salle, M. Chem. Soc. Rev. 2011, 40, 30.

[4] Fernandez-Herrera, M. A.; Lopez-Munoz, H.; Hernandez-Vazquez, J. M. V.; Sanchez-Sanchez, L.; Escobar-Sanchez, M. L.; Pinto, B. M.; Sandoval-Ramirez, J. Eur. J. Med. Chem. 2012, 54, 721.

[5] Leblond, J.; Petitjean, A. Chem. Phys. Chem. 2011, 12, 1043.

[6] Ghodratbeigi, M.; Rashidi-Ranjbar, P.; Abbasi, A. J. Mol. Struct. 2011, $990,140$.

[7] Klarner, F. G.; Kahlert, B. Acc. Chem. Res. 2003, 36, 919.

[8] Kihel, L. E.; Clement, M.; Bazin, M. A.; Descamps, G.; Khalid, M.; Rault, S. Bioorg. Med. Chem. 2008, 16, 8737.

[9] Fernandez-Herrera, M. A.; Lopez-Munoz, H.; Hernandez-Vazquez, J. M. V.; Sanchez-Sanchez, L.; Escobar-Sanchez, M. L.; Pinto, B. M.; Sandoval-Ramirez, J. Eur. J. Med. Chem. 2012, 54, 721.

[10] Xiong, X. Q.; Cai, L.; Tang, Z. K. Chin. J. Org. Chem. 2012, 32, 1410 (in Chinese). (熊兴泉, 蔡雷, 唐忠科, 有机化学, 2012, 32, 1410.)

[11] Xiao, S. Y.; Zhu, J.; Mu, X. J.; Li, Z. H. Chin. J. Org. Chem. 2013, 33, 1668 (in Chinese).

(肖尚友, 朱俊, 穆小静, 李正华, 有机化学, 2013, 33, 1668.)

[12] Nguyen, H. H.; Kurth, M. J. Org. Lett. 2013, 15, 362.

[13] Troegel, B.; Lindel, T. Org. Lett. 2012, 14, 468

[14] Read, M. L.; Gundersen, L. L. J. Org. Chem. 2013, 78, 1311.

[15] Collados, J. F.; Toledano, E.; Guijarro, D.; Yus, M. J. Org. Chem. 2012, 77, 5744.

[16] Kandre, S.; Bhagat, P. R.; Sharma, R.; Gupte, A. Tetrahedron Lett. 2013, 54, 3526

[17] Mahindra, A.; Sharma, K. K.; Jain, R. Tetrahedron Lett. 2012, 53, 6931

[18] Kappe, C. O. Angew. Chem., Int. Ed. 2004, 43, 6250.

[19] Polshettiwar, V.; Varma, R. S. Acc. Chem. Res. 2008, 41, 629.

[20] Zhao, Z. G.; Shi, Z. C.; Liu, M.; Liu, X. L. Bioorg. Med. Chem. Lett. 2012, 22, 7730 .

[21] Li, G. H.; Shi, Z. C.; Li, X. R.; Zhao, Z. G. J. Chem. Res. 2011, 35, 278.

[22] Shi, Z. C.; Zhao, Z. G.; Liu, X. L.; Wu, L. L. J. Chem. Res. 2011, $35,198$.

[23] Dayal, B.; Speck, J.; Bagan, E.; Tint, G. S.; Salen, G. Steroids 1981, $37,239$.

(Cheng, F.) 